\title{
Editorial: Topical Collection of the Juno Mission Science Objectives, Instruments, and Implementation
}

\author{
S.J. Bolton ${ }^{1} \cdot$ J.E.P. Connerney ${ }^{2,3}$
}

Published online: 30 October 2017

(C) Springer Science+Business Media B.V. 2017

It seems every day brings news of the discovery of another extrasolar planetary system, as astronomical telescopes throughout (and orbiting) our own planet search relentlessly for them. And yet, conventional theories for solar system origin and evolution are challenged to explain these new planetary systems. Within our own solar system, missions to the outer planets (Pioneer, Voyager, Galileo, and Cassini) have raised questions that existing theories of planetary formation are ill prepared to answer. The Galileo probe sampled Jupiter's atmosphere and found it remarkably lacking in water abundance, a result inconsistent with prevailing ideas of solar system formation. The Juno mission was born out of the desire to reconcile these discoveries with our understanding of solar system formation, or to grow a new understanding of solar system origin and evolution. We understood that our own system's largest planet, Jupiter, represented the key piece of the puzzle in the quest to understand the history of Earth and the other planets in our solar system.

Thus the Juno Mission was conceived to understand the formation, evolution and internal structure of Jupiter. The Juno measurement objectives are: (1) determination of the higher harmonics of the planet's gravity and magnetic fields; (2) investigation of the convection that drives the general circulation; (3) determination of the global oxygen abundance and local variations in water and ammonia forced by meteorology; and (4) the exploration of Jupiter's auroral zones and the transfer of angular momentum (via magnetic coupling) to the Jovian nebula and satellites.

Juno's science goals and objectives are central to all three NASA Science Themes: EarthSun System, Solar System, and Universe. Juno's science objectives are both broad and

S.J. Bolton

sbolton@swri.edu

J.E.P. Connerney

jack.connerney@nasa.gov

1 Southwest Research Institute, San Antonio, TX, USA

2 Solar System Exploration Division, Planetary Magnetospheres Laboratory, NASA Goddard Space Flight Center, Greenbelt, MD 20771, USA

3 Space Research Corporation, Annapolis, MD, USA 
ambitious. Juno peers deep inside Jupiter to probe the interior and deep atmosphere; Juno also looks outward upon its luminous aurora and polar magnetosphere. These objectives have been identified in both the National Academy's Solar System Exploration Decadal Survey and in its Sun-Earth Connections Decadal Survey. The National Academy's Astronomy and Astrophysics Decadal Survey identified the study of star formation, and the formation of planetary systems, as well as giant and terrestrial planet birth and evolution as high priority. Juno constitutes a major step forward in achieving each of these high priority science objectives.

Juno is an extraordinary mission of discovery and exploration. Peering down through the clouds and deep into Jupiter's atmosphere, Juno reveals fundamental processes of the formation and early evolution of our solar system. Contemporary theories of solar system origin and evolution are at an impasse, one that Juno observations are designed to overcome. Truly, the history of our solar system is found in the formation of the planet Jupiter. The stakes are even higher. Planetary systems like ours may be required for life to originate and evolve. How common are such systems? Juno supplies the critical information needed to understand the formation of our own solar system, and perhaps extra-solar planetary systems, and the conditions needed to form terrestrial-type planets.

Solar system formation models all begin with the collapse of a proto-solar nebula. Because Jupiter is mostly hydrogen and helium, it must have formed early, while the proto-solar nebula was still present. Being much more massive than the other planets, Jupiter probably grew more rapidly than any other planet, perhaps in the first few to $10 \mathrm{Myr}$; certainly before the gaseous accretion disk dissipated. How this happened, however, is unclear.

One set of models proposes that a protoplanetary core ( $\sim 10$ or more Earth masses $)$ was formed first, by accretion in the cold outer part of the proto-solar nebula in a span of $\sim 2$ to 5 million years. The collapse of the surrounding hydrogen and helium followed, yielding a planet with a central dense core of at least 10 Earth masses (ME) within a hydrogen/helium outer envelope. An alternate model proposes an even more rapid process: a gravitational instability in the nebula triggers a collapse that forms the giant planets in about $0.1 \mathrm{Myr}$, resulting in a core of less than six Earth masses — and perhaps none.

Differences between these scenarios are profound. Even more importantly, the role of icy planetesimals in planetary formation hangs in the balance-and with them, the origin of Earth and other terrestrial planets. The role of icy planetesimals-likely carriers of volatiles, including water and organics that are the fundamental building blocks of life and progenitors of bio-molecules on early Earth-remains particularly crucial. Juno measures water abundance and determines if Jupiter has a core, directly resolving the origin of this giant planet and by inference the solar system. In addition, by mapping its gravitation and magnetic fields, Juno reveals Jupiter's interior structure and measures the mass of its core.

How deep Jupiter's zones, belts, and other features penetrate is one of the most fundamental outstanding questions in Jovian atmospheric dynamics. By mapping global variations in atmospheric composition, temperature, cloud opacity and dynamics to depths much greater than 100 bars, Juno determines the structure and dynamics of Jupiter's atmosphere below the cloud tops for the first time.

The mission design most advantageous to the study of Jupiter's interior-within the practical realities of orbiting spacecraft and orbital dynamics-is one in which the spacecraft orbits from pole to pole in close proximity to the planet. Of necessity, this orbit is highly elliptical, carrying Juno and its science instruments throughout Jupiter's voluminous magnetosphere. This orbit is also ideal for studies of the polar magnetosphere and aurora, a science objective first identified ("Jupiter Polar Orbiter") in the 1980's after the remarkably 
successful Voyager flybys. Thus with the addition of a full complement of fields and particles instrumentation, along with UV and IR spectrographs, Juno could service another set of outstanding science objectives concerning the polar magnetosphere and aurora.

Jupiter's powerful magnetospheric dynamics create the most intense aurora in our solar system, via energetic electron and ion collisions in its upper atmosphere. No spacecraft prior to Juno ventured so close to Jupiter in transit across the polar magnetosphere, so our only tools for understanding Jovian aurora were those of remote imagers. In contrast, Juno directly measures the distributions of these charged particles, their associated fields, as it images the contemporaneous ultraviolet and infrared emissions of the polar magnetosphere. This greatly improves our understanding of one of the most remarkable phenomena of our solar system.

Juno's technical approach is synergistic with the science requirements and the practicalities of spaceflight. The mission design, spacecraft design, and science observation plan work together within a simple and robust operational concept. Juno uses a spinning, solar-powered spacecraft in a highly elliptical polar orbit to avoid the most intense of Jupiter's high radiation environments. The initial article in this special collection provides an overview of Juno's scientific themes (origin, magnetosphere, interior and atmosphere) as well as the mission design and Juno science operations center (JSOC). Juno's scientific payload is described in the remaining articles and includes: a dual frequency gravity/radio science system (X\&Ka band up/X\&Ka band down); a six-wavelength microwave radiometer for atmospheric sounding and composition; magnetometer sensor suite complete with star cameras for accurate attitude determination; multiple plasma and energetic particle detectors; a radio/plasma wave experiment; an ultraviolet imager/spectrograph; an infrared imager/spectrometer; and a fourcolor camera (JunoCam) to provide the public with their first glimpse of Jupiter's poles. An additional article describes the Juno radiation monitoring investigation.

This Topical Collection provides the rationale for the Juno Mission and a set of science objectives to be achieved by mission's end - a plunge into Jupiter's atmosphere in the interests of planetary protection (mainly Europa). A good many science orbits ( $>32$ ) will have been achieved before then, wrapping the planet in a dense net of observations optimized for characterizing Jupiter's potential fields. The Collection also serves as an indispensable reference for the mission plan and the instruments that make up Juno's scientific payload, describing in great detail the development, calibration, and operation of the tools that Juno uses to make its measurements. It is by any measure an impressive extension of the tools of scientific analysis to another world. 